\title{
Researching experiences in a cohort program to influence transfer self-efficacy
}

\author{
Laura A. Wood ${ }^{1}$, Angela J. Little ${ }^{1}$, Vashti Sawtelle ${ }^{2,1}$ \\ ${ }^{I}$ Physics \& Astronomy Department, Michigan State University, 220 Trowbridge Rd, East Lansing, MI, 48824 \\ ${ }^{2}$ Lyman Briggs College, Michigan State University, 919 E Shaw Ln, East Lansing, MI, 48825
}

\begin{abstract}
There remains a great deal of research to do on improving the transfer experience for students transitioning from two-year colleges to four-year colleges. In this paper, we describe data collected from interviewing current students at a large Midwestern research university who are members of a cohort program which will be adapted for transfer students to join. This cohort program is designed to give students - intending to major in the natural sciences, and predominantly from underrepresented backgrounds - support in academics, research experiences, and the social experience of integrating into the university. The interview protocol elicited discussion of these students' self-efficacy to complete their science degrees, navigate the academic requirements, and continue in their chosen life paths, specifically drawing out mastery, vicarious learning, and social persuasion experiences. We will discuss how student experience in the cohort program may support developing self-efficacy in the transfer process.
\end{abstract}

\section{I.INTRODUCTION}

A number of equity-related programs exist in college physics and the natural sciences with the goal of knitting students into a community where they receive holistic and academic support. Indeed, the recent Phys 21 taskforce report recommends creating such programs [1].

These programs can be said to build a cohort of students. In this paper, we present analysis using a coding scheme for self-efficacy or "confidence in one's ability to perform a particular task" [2]. A reason for focusing on self-efficacy is that it is a strong predictor of retention and academic success [3]. By analyzing interviews with students both within and outside of a cohort, we argue that a cohort can provide opportunities for contributing to students' self-efficacy.

Specifically, our research focuses on a cohort for science majors, who are primarily students of color, at a large, predominantly white, Midwestern research university (LMRU). This cohort program will expand to include transfer students who come from regional two-year colleges (TYC). We will call the current cohort EC (established cohort) and the developing cohort TC (transfer cohort). Researching and developing the TC is particularly important, as limited research has been done on the experience of transfer students after transferring $[4,5]$.

We focus on understanding the first-year student experience in the EC, and the current transfer student experience at LMRU before the cohort expands. This paper uses comparative analysis of three student interviews - two with EC members, and one with a transfer student. Selfefficacy coding gave us insight into the differences between this transfer student's experiences and those in the formal cohort. We argue that a cohort experience affords students more opportunities to develop positive self-efficacy.

\section{II.COHORT DEFINITION}

We recognize that student descriptions of a cohort are not necessarily aligned with the way academics would define a cohort. Therefore, we analyze two things described by our student interviewees: (1) descriptions of the cohort and (2) evaluations of their self-efficacy due to cohort experiences. Thus, we need a definition of cohort as a starting point to determine what programs will fit our research question.

We are examining how the cohort experience is tied to students' evaluations of their self-efficacy to do well on academic tasks. Thus, we used inductive coding to analyze the student descriptions of the cohort. To define a cohort, we modified literature-based definitions. One definition of cohort is "a group of students or participants ... who proceed through a program of learning ...taking all of their courses or instruction in a sequential manner" [6]. Similar definitions are found commonly in literature, but not always using the word cohort (for example learning communities) $[7,8]$.

Cohorts are also defined as having certain goals, like social and academic integration, interpersonal relationships with faculty and peers, and academic involvement [6-10]. Thus, we turn to the goals of a cohort to further our definition and broaden it to include programs in which not all courses are taken together. In our work, we define a cohort as a program with two main attributes.

1) Builds social and academic connections, and places students in some shared courses.

2) Targets students in similar college positions (e.g., grade-level, major, transfer students).

It might appear that a cohort could be any set of experiences with these goals, but we find that participation in a formal cohort is more than a set of individual aspects.

\section{III.RESEARCH METHODS}

Our research questions (RQ) are the following: (RQ1) What contributes to cohort members and transfer students developing self-efficacy? (1a) What are students' evaluations of their self-efficacy? (1b) What experiences are they using to evaluate that? In particular, what cohort experiences are cohort members using? (2) In our ongoing work, we ask how that information might be used in preparing for the $\mathrm{TC}$, as this is a preliminary study in the context of a larger project. 


\section{A. Interview protocol development}

There are four types of contributing experiences for developing self-efficacy, three on which we focused in our interviews: mastery (past experiences which affect confidence), social persuasion (others' statements of one's ability), and vicarious learning experiences (judging one's ability based on how others perform) [2]. Descriptions of physiological state (emotional state) were not a goal of the interviews, as they are very different in form than the other contributing experiences.

Questions meant to elicit mastery experiences and general self-efficacy statements included: "How well prepared do you feel to tackle the challenges in completing your major?" Social persuasion questions included, "When you're making academic decisions, to whom do you talk?" Vicarious learning questions included, "How do you think being a part of this cohort has changed your college experience?" Question wording depended on if the interviewee was in a cohort or not.

There were also questions designed to elicit a description of the cohort experience. Questions included, "Can you describe what it looks like to be a part of the cohort?" Other questions in the full protocol used by multiple researchers were on mindset and sense of belonging. The interviews were semi-structured and about an hour long.

\section{B. Cohort and transfer interview participants}

In this paper, we compare the experiences of two EC members (not transfer students) and one transfer student (not in a cohort). Victoria is a first-year chemistry major in the EC. She has been interested in chemistry since childhood when she would play around with mixing "the chemicals under the sink." What she is worried about in completing her major is just how much there is to do for her chemistry major. She was fully convinced she wanted to "jump ship" and switch to chemical engineering, at her dad's suggestion, until she realized it wasn't a lighter workload and that "she wasn't a chemical engineer, she's a chemist." Instrumental in this decision was her discussion with the EC-specific advisor, Marie, who said she would support Victoria if she wanted to change majors but that she "didn't think it was [her]."

Shaina is a first-year, pre-med track, human biology major in the EC. Since high school, she has felt that math and science come easier to her than other subjects and likes that with math and science "there's always a way to get the answer." She chose to major in human biology upon advice that human biology is versatile. For example, her sister started out as a human biology major and smoothly switched to dental work. Shaina joined the EC after much discussion with a friend. She has appreciated being able to take many classes with people from the cohort and studying with them conveniently due to the shared cohort living space.

Amani is a transfer student in her first-year at LMRU, majoring in human biology in the pre-med track. She decided to start at a TYC after high school, mostly to save money, where she majored in biology and was a full-time student year-round. She appreciated getting to know her classmates and teachers well at the TYC, and in comparison, was "baffled" by class sizes of 400 people when she transferred. Amani is not in a cohort, but she had cohort-like experiences - largely from her participation in a Religious Association and a Medical Student Association. She takes advantage of informational pamphlets and emails for finding social events and volunteer opportunities, is involved in volunteer experiences, and is seeking a variety of internships.

\section{Coding scheme}

We coded the interviews for self-efficacy, in a deductive coding style [11]. This captures students' evaluations of their self-efficacy (RQ1a). The coding scheme highlights the four types of experiences that contribute to self-efficacy.

To describe students' experiences (RQ1b), we used an inductive coding method [11] to code the interviews for descriptions of the cohort. The cohort coding scheme consists of two codes: "description of cohort" and "cohort impact," with many sub-codes to capture the variety of responses. Self-efficacy coding that overlapped with "cohort impact" allowed us to draw connections between experiences in the cohort and the students' self-efficacy.

\section{IV.ANALYSIS}

Amani is proactive at finding many cohort-like resources. However, we see that while Amani finds them helpful, she makes few statements to suggest they impacted her selfefficacy. Contrasting her experiences with Shaina's and Victoria's, we find that the full cohort program provides them a more personalized and better academic experience, offering a range of opportunities to develop self-efficacy.

\section{A. Amani's cohort-like experience}

Amani finds a lot of internship and volunteer information herself, something the EC would provide. She thinks it was easy to sign up online to volunteer at a hospital, and she discovered other opportunities through Google, a local respite center, and the student center. She also tries to take advantage of study groups, but has some scheduling difficulty.

"[S]ome of the clubs that I've been to, they would ask me,

"What's your major?" ...So then we would ...study

together. And then in some of the classes, they would form study groups...but I didn't go on a regular basis. [I]t's been tricky to find the time to go to study groups."

Amani finds it difficult to schedule class-based study groups. While clubs, in contrast, help her find study partners, she does not make a self-efficacy statement about them.

Amani is part of a few clubs she found through an oncampus informational event, as well as through paying attention to postings and newsletters. She is most active in the Religious Association, and said, "There were a few 
people... We had similar classes that we were taking. So through that, we connected a lot, too." Being a part of the Medical Student Association also provided some cohort-like opportunities. Amani said, "through [both groups], they helped me find really good volunteer options, too. I was really appreciative of that. [T] hey gave us tips and ideas for studying for the MCAT... I was really grateful for that. They have really good clubs here."

We can see that Amani is finding her own ways of making social connections and getting academic help and opportunities, all experiences the EC aims to foster. However, while such experiences seem important to her, she does not make many positive self-efficacy statements.

\section{B. Personalized experience in cohort}

Shaina and Victoria describe similar positive experiences to Amani in the EC, like finding friends and social events, and forming study groups, but they also describe benefits of the cohort beyond those. In this section, we will compare two strikingly similar experiences described by Amani and Shaina or Victoria and see how the full cohort experience offers more self-efficacy opportunities. Self-efficacy statements are italicized in the following transcript excerpts.

Amani recounts overcoming negative self-efficacy from hearing motivational speakers at new student orientation.

"Well, coming here [to LMRU]..., I was really nervous about it. But it's been, overall, a really great experience, and like I said, I failed that one class at [TYC] which really brought my confidence really down. But coming here during orientation, I remember they were talking about, 'You're going to have a lot of falls, and you might not pass every single class.... as long as you focus on the right path and where you want to go, it'll work itself out and everything.' That really spoke to me because I was like, 'Yeah. I just failed this class. And I'm really worried about it. But hopefully, it does all work itself out.",

Amani found this reassuring ("that really spoke to me;"), but does not make a positive self-efficacy statement.

In contrast, a motivational speaker who came to speak to the EC completely changed Shaina's view of potential career paths. The speaker told a story about how her son was able to get a job at Google without perfect grades. The interviewer asked what Shaina would have responded prior to the talk "if someone had asked, "Can you work at Google?" Shaina said, "Absolutely not." If asked after the talk, she'd say, "If I work at it, yes, if that's the path I want to take I think for sure I could take it." Her description of the seminar speech is coded under "description of cohort," and she attributes her career self-efficacy directly to that experience. Shaina particularly found it important that the speaker was "close to the [EC] family," saying, "it's not somebody that was posting about an article or something online talking about 'contact me' and then they never heard back." Shaina shows us how the cohort impacted her self-efficacy through a personally relatable experience.

Another experience shared by all three participants is making a four-year course schedule. Victoria and Shaina describe EC staff member Marie helping them make schedules and that allowing them to visualize their capability of completing their majors. Amani did this on her own, and it did impact her self-efficacy.

"Well, I wrote out everything that I had to take and when I wanted to take it. And I think that since I did take a lot of classes in the summer, that helped me get ahead of everything. ...So in [class scheduling], I'm like not stressing about. Mostly just that I pass with good grades, that's the biggest stressor."

It seems likely that the act of making a schedule to visualize ability to complete a degree in four years is an experience that positively contributes to self-efficacy whether or not a student is in a cohort.

However, we still see clearer self-efficacy statements when Victoria describes Marie creating her four-year schedule. In contrast to Amani's vague self-efficacy statement about stress, Victoria makes clear, easy-to-code vicarious learning and social persuasion statements, saying,

"We had an advising meeting. ...After that [Marie] lays out your four-year plan.... And after she put everything on this little schedule I could visualize like, oh, this is possible. And they're ...really for you for your success...

So they're like, 'No, you can definitely do it. There's all these resources you can use if you're having trouble in your classes.' ...For me, I was like, 'Okay, it's been done, someone else is doing it. I can do this, it's possible here."

Seeing a feasible four-year plan ("description of cohort") is not the only thing contributing to Victoria's self-efficacy here, but also that she is part of a supportive community of faculty and similarly situated learners.

The cohort provides more personalized experiences for Shaina and Victoria than the cohort-like aspects Amani finds on her own. The personally relatable experience of a cohort that is like a supportive family provides Shaina and Victoria chances to develop their self-efficacy through vicarious learning and social persuasion opportunities. This is one way the cohort is more than a sum of its parts.

\section{Good academic experience in cohort}

Another theme in Shaina's and Victoria's interviews is the positive impact the teaching practices used in the cohort had on their academic self-efficacy. In the cohort, academic coaching involves a student or staff member working with small groups of students taking the same class. They work on problems together and review difficult material in an interactive way. Outside the cohort, students experience "bafflingly" large classes of up to 400 students (as Amani described), teaching that only mentions test topics once or twice (Amani), a tutoring center where "they're just trying to get you in there, get you out of there, get it done" (Victoria), 
difficulties getting to know peers (Amani), and difficulties scheduling study groups (Amani). Shaina and Victoria report very different academic experiences as part of the cohort.

Victoria did not just find Marie instrumental in bolstering her degree self-efficacy. She also attributes staying in the major to the cohort's integration of social and academic life, in a quote in which the "cohort impact" code (bolded here) exactly overlaps with self-efficacy statements.

"That's the reason - I know I have like someone that I can go do my homework with every night. No offense to [LMRU's tutoring] center, ...that's not my favorite place to go either. Honestly, having [EC], having [Malcolm] is like a major part of my success in my math classes. ...I cannot tell you if I would still be in the [major] at this point; like I might have been a communications major a long time ago."

There are two aspects of the cohort that Victoria describes impacting her persistence: the cohort studying community and the academic coach Malcolm. These features are both salient in interviews with cohort members. Shaina describes the ease of group studying in the EC space, saying,

"There's tables in there and all of the tables will be in the same subject so you'll ... be like, 'oh you're studying right now? Let me ...study with them.' That's how I interact with the space is coming out and ...finding other people in the same classes and sitting down and studying."

Shaina attributes success on a test to preparing more by studying with cohort members and EC-specific academic coaches. "I did better on the first exam than on the second exam because I did more outside of the classroom for the first exam than for the second. ...There's ...a student like us .... and she goes and she teaches in the [EC room] to a whole bunch of students who are in it right now."

In this way, the cohort consists of good teaching experiences that mitigate difficulties students face finding study groups and smaller communities within big classes.

\section{V.DISCUSSION}

These cases show that two students in the EC have experiences that support stronger self-efficacy statements than a transfer student who experienced cohort-like aspects. Shaina and Victoria make clear academic self-efficacy statements about the cohort's good teaching practices and also attribute positive self-efficacy to vicarious learning and social persuasion opportunities in the personalized cohort.

A limitation of our claims is that the interviewer may not have probed self-efficacy in the same way in Amani's interview. However, the main questions were always asked in similar ways, and since there is a lack of strong positive self-efficacy statements across Amani's entire interview, we feel confident that our claims characterize a meaningful difference between a cohort and a non-cohort experience.

We do not yet know what the TC will look like and may need to continue to refine what a "cohort experience" entails. For example, EC members take a seminar course in the first and second years and also mostly live together. Transfer students may be more likely to be commuters, and the seminar course may also need to be changed.

Although this paper does not suggest exactly how the EC should change to accommodate transfer students, it indicates that the cohort might be beneficial to transfer students. As the EC expands to include transfer students, Amani's analysis suggests that she would likely benefit from the full cohort experience: hearing relatable motivational speakers in the seminar, studying in a shared cohort space with other similarly situated students, having access to cohort-specific advisors and academic coaches, etc. In the interviews, students also describe the effects of the cohort on their sense of belonging, identity, and social connections. There is more work to be done on researching the cohort's self-efficacy impact, but also many other effects of the cohort to examine.

\section{ACKNOWLEDGEMENTS}

We gratefully acknowledge the work done by Abigail Green in developing the self-efficacy coding scheme. We are deeply indebted to the interviewees who shared their experiences with us. This work was supported in part by National Science Foundation grant \#1742381.

[6] D. W. Rausch and E. Crawford, Metropolitan Universities, 23(1), 79-89 (2012).

[7] M. L. A. Stassen, Research in Higher Education, 44(5), 581-613 (2003).

[8] V. Tinto, Higher Education Monograph Series, 1, 1-8 (2003).

[9] L. R. M. Hausmann, et al., Research in Higher Education, 48(7), 803-839 (2007).

[10] J. M. Braxton, J. F. Milem, and A. S. Sullivan, The Journal of Higher Education, $71(5), 569$ (2000).

[11]P. Leavy, The Oxford handbook of qualitative research (Oxford: Oxford University Press, 2015), ch. 28 